\title{
The association between alcohol use and suicide attempt in employees
}

\author{
Won Young Choo ${ }^{1}$, Ung Lee ${ }^{1}$, Jae-Hyun Park ${ }^{2}$, Young-Chul Shin ${ }^{1,3}$, Mikyung Sim ${ }^{3}$, \\ Kang-Seob Oh ${ }^{1}$, Dong-Won Shin ${ }^{1}$, Sang-Won Jeon ${ }^{1,3}$, Min-Kyoung Kim ${ }^{4}$, \\ Sung Joon $\mathrm{Cho}^{1,3}$
}

\begin{abstract}
${ }^{1}$ Department of Psychiatry, Kangbuk Samsung Hospital, Sungkyunkwan University School of Medicine, Seoul, Korea
${ }^{2}$ Department of Social and Preventive Medicine, Sungkyunkwan University School of Medicine, Suwon, Korea

${ }^{3}$ Workplace Mental Health Institute, Kangbuk Samsung Hospital, Sungkyunkwan University School of Medicine, Seoul, Korea

${ }^{4}$ Department of Psychiatry, CHA Ilsan Medical Center, CHA University School of Medicine, Goyang, Korea
\end{abstract}

Received: March 5, 2021

Revised: May 3, 2021

Accepted: May 4, 2021

Corresponding author:

Sung Joon Cho

Department of Psychiatry, Kangbuk Samsung Hospital,

Sungkyunkwan University

School of Medicine, 29

Saemunan-ro, Jongno-gu,

Seoul 03181, Korea

Tel: +82-2-2001-2214.

E-mail: sjcho0812@hanmail.net

\section{ABSTRACT}

Purpose: This study aimed to analyze the alcohol use patterns that may influence suicide attempt in employees who have suicidal ideation.

Methods: The 15,199 participants were classified into three groups according to suicide risk severity. Participant scores on the Alcohol Use Disorders Identification Test-Korea (AUDIT-K), subscale for drinking amount/frequency (AUDIT-C, items 1-3), subscale for dependence/related problems (AUDIT-D/P, items 4-10), and other sociodemographic and psychiatric scales were analyzed between the groups using one-way analysis of variance (ANOVA) and the chi-square test, followed by post hoc analysis using the Bonferroni correction.

Results: A statistically significant difference between all three groups was observed for AUDIT-D/P scores after post hoc analysis $(P<0.001)$, indicating an increasing trend for greater suicide risk. This trend was present in both sexes and across all age groups above 30 years old.

Conclusion: Alcohol dependence/related problems are significantly associated with suicide attempt among employees. Assessing and preventing suicide risk according to these issues may minimize socioeconomic losses due to suicide.

Keywords: Alcohols; Employee; Suicide
This is an Open Access article distributed under the terms of the Creative Commons Attribution Non-Commercial License (https:// creativecommons.org/licenses/ by-nc/4.0/).

\section{INTRODUCTION}

According to Statistics Korea, Korea's suicide rate was 26.9 per 100,000 individuals in 2019 [1]. Korea has the highest suicide rate and average number of suicides among Organisation for Economic Co-operation and Development (OECD) countries [2]. Based on the data from the National Health Insurance Policy Research Institute of Korea National Health Insurance Service, the socioeconomic cost of suicide is approximately 6.5 trillion won and it is the third high- 
est economic impact factor after cancer and cerebrovascular disease among major causes of death in Korea [3]. Moreover, the actual socioeconomic cost of suicide is assumed to be significantly greater considering the medical expenses for injuries, the consequences of attempted suicides, and the physical and psychological treatment for the bereaved. Therefore, assessing the risk of suicide in adults in the working population can prevent staggering losses for the country, corporations, and society. Thus, works that explore the influencing factors and preventative measures for suicide are needed.

Meanwhile, the annual drinking rate of Koreans aged 19 years and older in a standardized population was $79.7 \%$, according to the 2018 Korea National Health and Nutrition Examination Survey (KNHANES), conducted by the Ministry of Health and Welfare [4]. According to 2017 data from the World Health Organization (WHO), this rate is approximately two times higher than the average annual drinking rate in the global population of people who are 15 years and older [5]. In a previous autopsy study conducted by the Korean National Forensic Service, the blood alcohol concentration (BAC) of $48.4 \%$ of people who committed suicide between 2007 and 2009 was over 0.05 at the time of committing suicide [6], which is commonly known as the global standard for driver's license suspension limit. Alcohol consumption affects perception, judgement, and memory function by weakening emotional control and inducing impulsive aggression and cognitive distortion [7]. Hence, alcohol consumption in people who are at imminent risk of suicide plays a crucial role in unplanned suicide attempts [8], while acute alcohol intoxication is known to provoke suicidal ideation and suicide attempts in at-risk people [9]. Also, consuming alcohol after a suicide attempt that had serious medical consequences increases the risk of re-attempting suicide or having a successful suicide [10]. Drinking is therefore considered a predisposing factor for suicide. Behavioral problems due to impulsiveness after drinking are presumed to be directly related to suicidal ideation and induce actual suicide attempts in addition to other problems related to drinking. Several studies have explored the correlation between drinking and suicide, but no study has yet investigated how an individual's drinking behavior is associated with suicide attempts.

This study aimed to examine the link between factors related to drinking and attempted suicide in the working population by examining the effects of alcohol consumption in people who have suicidal ideation, cross-sectionally. This work may help to prevent future suicides of workers by eval- uating risks associated with suicide attempts and thus minimize socioeconomic losses of society. Correlations between drinking and suicide were analyzed according to sex and age, and other sociodemographic factors that might influence suicide attempts were also inspected.

\section{METHODS}

\section{Participants}

This was a cross-sectional study that targeted individuals between the ages of 20 and 65 who participated in a mental health examination program conducted by the Workplace Mental Health Institute of Kangbuk Samsung Hospital. The participants were employees of 52 different corporations, government institutions, and private institutions, who voluntarily participated in the study. This study was conducted from 2014 to 2019: a total of 15,226 individuals completed the survey on suicidal ideation and suicide attempt. After excluding 27 inappropriate responses in which answered that they attempted suicide without suicidal ideation, a total of 15,199 participants were included in the analysis. The 12,177 participants who had no suicidal ideation or suicide attempts were categorized into Group 1, while 2,843 participants who had suicidal ideation but no suicide attempts were categorized into Group 2, and 179 participants who had both suicidal ideation and suicide attempts were categorized into Group 3 (Fig. 1). The total missing data value was less than $1 \%$ for each variable. This study obtained the approval of the Institutional Review Board of Kangbuk Samsung Hospital (IRB no. KBSMC 2019-01-042). Informed consents were not obtained because the study was conducted using anonymized data only.

\section{Research tool \\ Sociodemographic assessment of participants}

Participants' age, sex, obesity index, marital status, education level, and work position were evaluated. The obesity index was assessed based on body mass index (BMI). Marital status was classified into single, married, or other. Education level was classified into high school/junior college or college degree or higher. Work position was classified into employee, manager, deputy section chief, section chief, deputy department head, department head, executive, or other.

\section{Major questionnaires}

To assess suicidal ideation, participants were asked to answer "never," "occasionally," or "almost always" to the ques- 


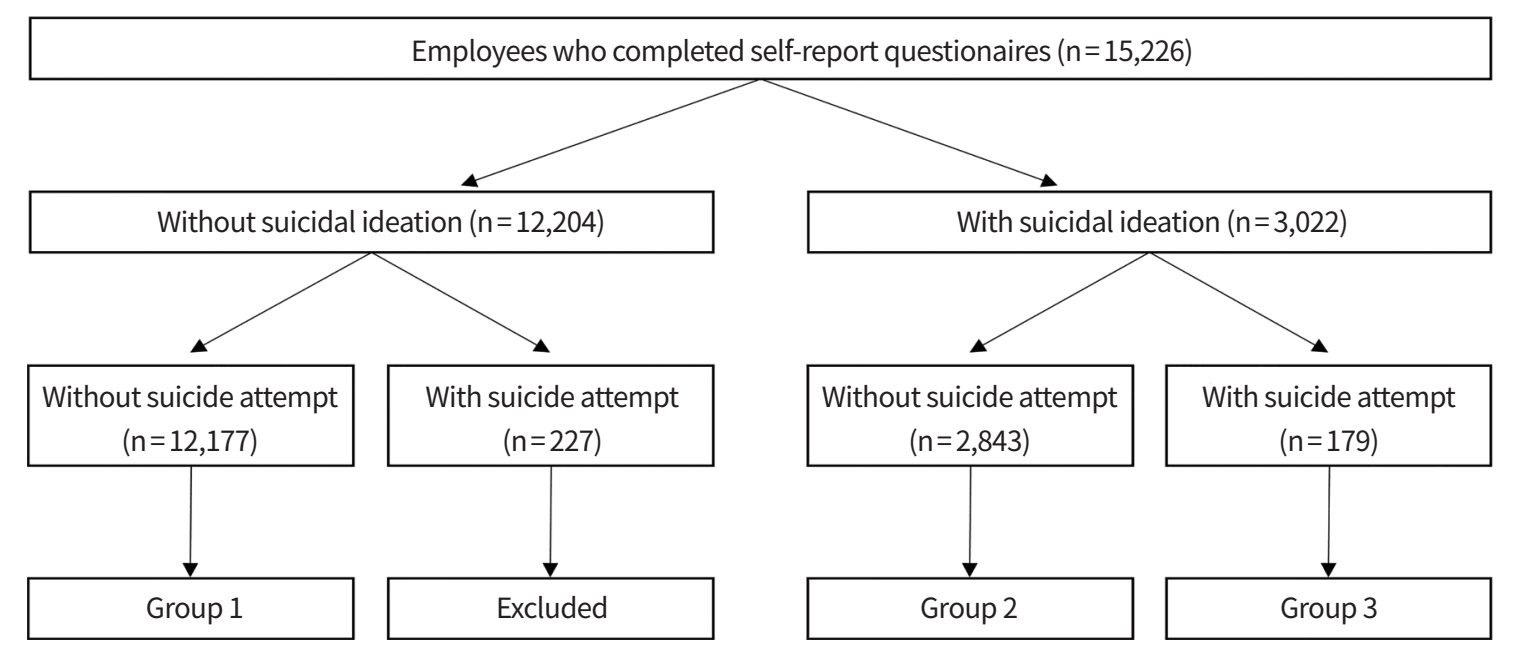

Fig. 1. Study population.

tion, "Have you thought about suicide in the past year?" from KNHANES [4]. Participants who answered, "never" were categorized into the "without suicidal ideation" group, while those who answered "occasionally" or "almost always" were categorized into the "with suicidal ideation" group.

To assess prior suicide attempt, participants were asked to answer "never," "self-injury but no suicide attempt," or "attempted suicide," to the question, "Have you attempted suicide in the past year?" from KNHANES [4]. Participants who answered "never" were categorized into the "without suicide attempt" group, while those who answered "self-injury but no suicide attempt" (133 participants) or "attempted suicide" (46 participants) were categorized into the "with suicide attempt" group in a broad sense, considering the strong linkage between nonsuicidal self-injury and suicide attempt [11, 12].

Drinking level was measured using the Alcohol Use Disorders Identification Test-Korea (AUDIT-K). Each item of the AUDIT-K evaluates consumption quantity, behavior, and presence of psychological-social problems [13]. The AUDIT-K consists of 10 items scored from 0 to 4 in which a high score indicates greater severity. The total score ranges from 0 to 40 . Items 1-3 were defined in previous studies as AUDIT-Alcohol Consumption Questions (AUDIT-C) [14], and their validity and reliability have been proven as a screening tool [15]. In this study, items 4-10, which relate to dependence and problematic behavior, have been defined as Alcohol Dependence/related Problem Questions (AUDIT-D/P).

\section{Other questionnaires}

The Korean version of the Center for Epidemiological Studies
Depression Scale (CES-D), developed for an epidemiological survey on depression in the general population, was used to assess depression symptoms [16]. The CES-D is a self-reporting scale consisting of 20 items in which a high score indicates greater severity. The total score ranges from 0 to 60 .

Anxiety symptoms were assessed using the Korean version of the Beck Anxiety Inventory (BAI) [17]. The BAl is a self-reporting scale consisting of 21 items scored from 0 to 3 in which a high score indicates greater severity. The total score ranges from 0 to 63 .

Occupational stress was measured using the Korean Occupational Stress Scale (KOSS) [18]. This scale consists of 27 items in seven subcategories: job demands, insufficient job control, interpersonal conflict, job insecurity, organizational system, lack of reward, and organizational culture. Each item is scored from 1 to 4 . The total score ranges from 27 to 108.

Several additional tools, including the Perceived Stress Scale (PSS) [19], Daily Life Stressors Scale (DLSS) [20], Connor-Davidson Resilience Scale (CD-RISC) [21], and World Health Organization-Quality of Life (WHO-QOL) scale [22], were used to assess different types of stress and quality of life.

Each scale was completed in a self-reporting format.

\section{Statistical analyses}

Data were analyzed using SPSS Statistics for Windows version 18.0 (SPSS Inc., Chicago, IL, USA). Participants were divided into three groups: participants without suicidal ideation and suicide attempt (Group 1), participants with suicidal ideation but without suicide attempt (Group 2), and participants with suicidal ideation and suicide attempt (Group 3). Multiple logistic regression model was used to evaluate the 
multicollinearity of variants such as depression, anxiety, occupational stress, alcohol, perceived stress, daily-life stresssors, resilience, quality of life. The model was statistically significant $(P<0.01)$. For further analysis, chi-square tests and one-way analysis of variance (ANOVA) tests were performed to analyze the sociodemographic and clinical characteristics of each group. Bonferroni corrections were performed to compare the three groups. The significance level for all statistics was established at $\mathrm{P}<0.05$.

\section{RESULTS}

\section{Sociodemographic characteristics}

The average age of the 15,199 participants was 39 years; $58.2 \%$ $(n=8,843)$ were male and $41.8 \%(n=6,356)$ were female.

Among the 15,199 participants, 3,022 participants had suicidal ideation and 179 participants had attempted suicide.
The severity of suicide risk increased from Group 1 to Group 3 by order. Increased suicide risk was significantly associated with decreased age $(P<0.001)$, female $(P<0.001)$, unmarried status $(P<0.001)$, lower education level $(P<0.001)$, and lower position such as employee or in a managerial position $(\mathrm{P}<$ $0.001)$. BMI was statistically different among the groups $(P=$ 0.029), but no apparent tendency was observed from Group 1 through Group 3 (Table 1).

\section{Psychiatric characteristics}

Several associations with self-reporting scales for increased suicide severity were observed. Scores for depression (CES-D), anxiety (BAI), occupational stress (KOSS), drinking (AUDIT-K), perceived stress (PSS), and daily-life stressors (DLSS) were significantly higher in Group 2 than Group 1, and higher in Group 3 than Group $2(P<0.001)$, while resilience (CD-RISC) and quality of life (WHO-QOL) were lower in Group 2 than Group 1 and

Table 1. Sociodemographic characteristics of employees

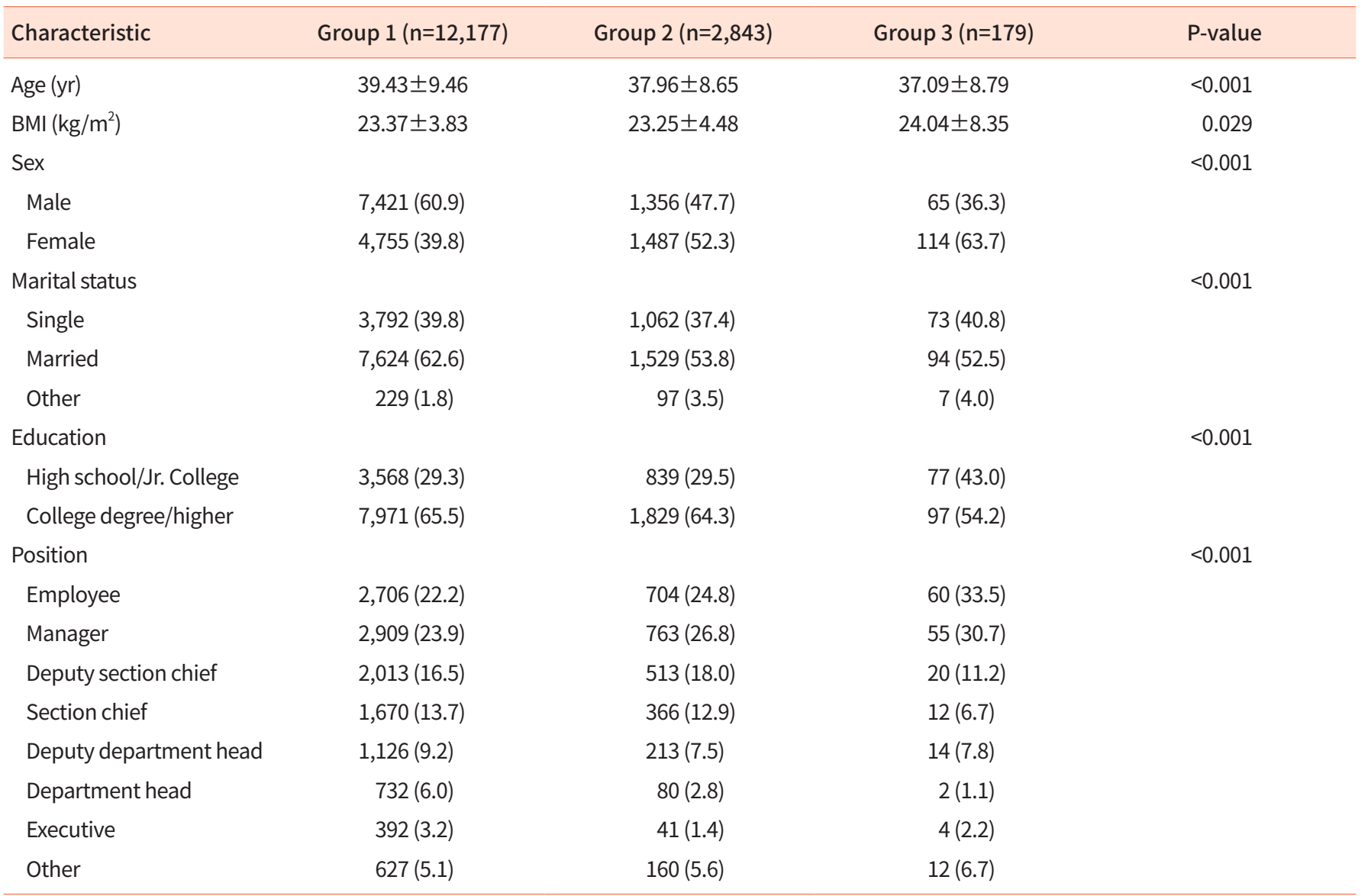

Values are presented as mean \pm standard deviation or number (\%). Group 1: participants who had no suicidal ideation and had not attempted suicide; Group 2: participants who had suicidal ideation and had not attempted suicide; Group 3: participants who had suicidal ideation and had attempted suicide.

BMI, body mass index. 
lower in Group 3 than Group $2(\mathrm{P}<0.001)$. These tendencies were mostly significant in post hoc analyses as well, yet no sta- tistically significant difference for daily-life stressors was observed between Group 2 and Group 3 (Table 2).

Table 2. Psychiatric characteristics of employees

\begin{tabular}{|c|c|c|c|c|c|c|c|}
\hline Characteristic & $\begin{array}{c}\text { Group } 1 \\
(n=12,177)\end{array}$ & $\begin{array}{c}\text { Group } 2 \\
(n=2,843)\end{array}$ & $\begin{array}{l}\text { Group } 3 \\
(n=179)\end{array}$ & P-value & $\begin{array}{c}\text { Group 1\&2 } \\
\text { P-value }^{\text {a) }}\end{array}$ & $\begin{array}{c}\text { Group } 2 \& 3 \\
\text { P-value }\end{array}$ & $\begin{array}{c}\text { Group } 1 \& 3 \\
\text { P-value }\end{array}$ \\
\hline Depression (CES-D) & $8.15 \pm 7.865$ & $17.57 \pm 11.587$ & $24.55 \pm 13.991$ & $<0.001$ & $<0.001$ & $<0.001$ & $<0.001$ \\
\hline Anxiety (BAI) & $5.48 \pm 6.574$ & $12.89 \pm 10.115$ & $20.72 \pm 13.021$ & $<0.001$ & $<0.001$ & $<0.001$ & $<0.001$ \\
\hline Occupational stress (KOSS) & $60.23 \pm 9.216$ & $66.07 \pm 9.370$ & $68.77 \pm 9.967$ & $<0.001$ & $<0.001$ & $<0.001$ & $<0.001$ \\
\hline Alcohol (AUDIT-K) & $7.77 \pm 6.290$ & $9.08 \pm 7.348$ & $11.22 \pm 8.227$ & $<0.001$ & $<0.001$ & $<0.001$ & $<0.001$ \\
\hline Perceived stress (PSS) & $15.66 \pm 5.262$ & $20.39 \pm 5.328$ & $22.63 \pm 5.722$ & $<0.001$ & $<0.001$ & $<0.001$ & $<0.001$ \\
\hline Daily-life stressors (DLSS) & $87.00 \pm 33.632$ & $99.17 \pm 13.402$ & $100 \pm 0$ & $<0.001$ & $<0.001$ & 1.000 & $<0.001$ \\
\hline Resilience (CD-RISC) & $65.53 \pm 15.222$ & $54.84 \pm 15.558$ & $50.64 \pm 17.664$ & $<0.001$ & $<0.001$ & 0.001 & $<0.001$ \\
\hline QOL (WHO-QOL) & $55.88 \pm 8.300$ & $48.06 \pm 7.963$ & $44.64 \pm 7.778$ & $<0.001$ & $<0.001$ & $<0.001$ & $<0.001$ \\
\hline
\end{tabular}

Values are presented as mean \pm standard deviation. Group 1: participants who had no suicidal ideation and had not attempted suicide; Group 2: participants who had suicidal ideation and had not attempted suicide; Group 3: participants who had suicidal ideation and had attempted suicide.

CES-D, The Center for Epidemiologic Studies Depression Scale; BAI, Beck Anxiety Inventory; KOSS, Korean Occupational Stress Scale; AUDIT-K, Alcohol Use Disorders Identification Test-Korea; PSS, Perceived Stress Scale; DLSS, Daily Life Stressors Scale; CD-RISC, The Connor-Davidson Resilience Scale; WHO-QOL, World Health Organization-Quality of Life.

a) Bonferroni correction.

Table 3. AUDIT-K specific factor analysis by sex

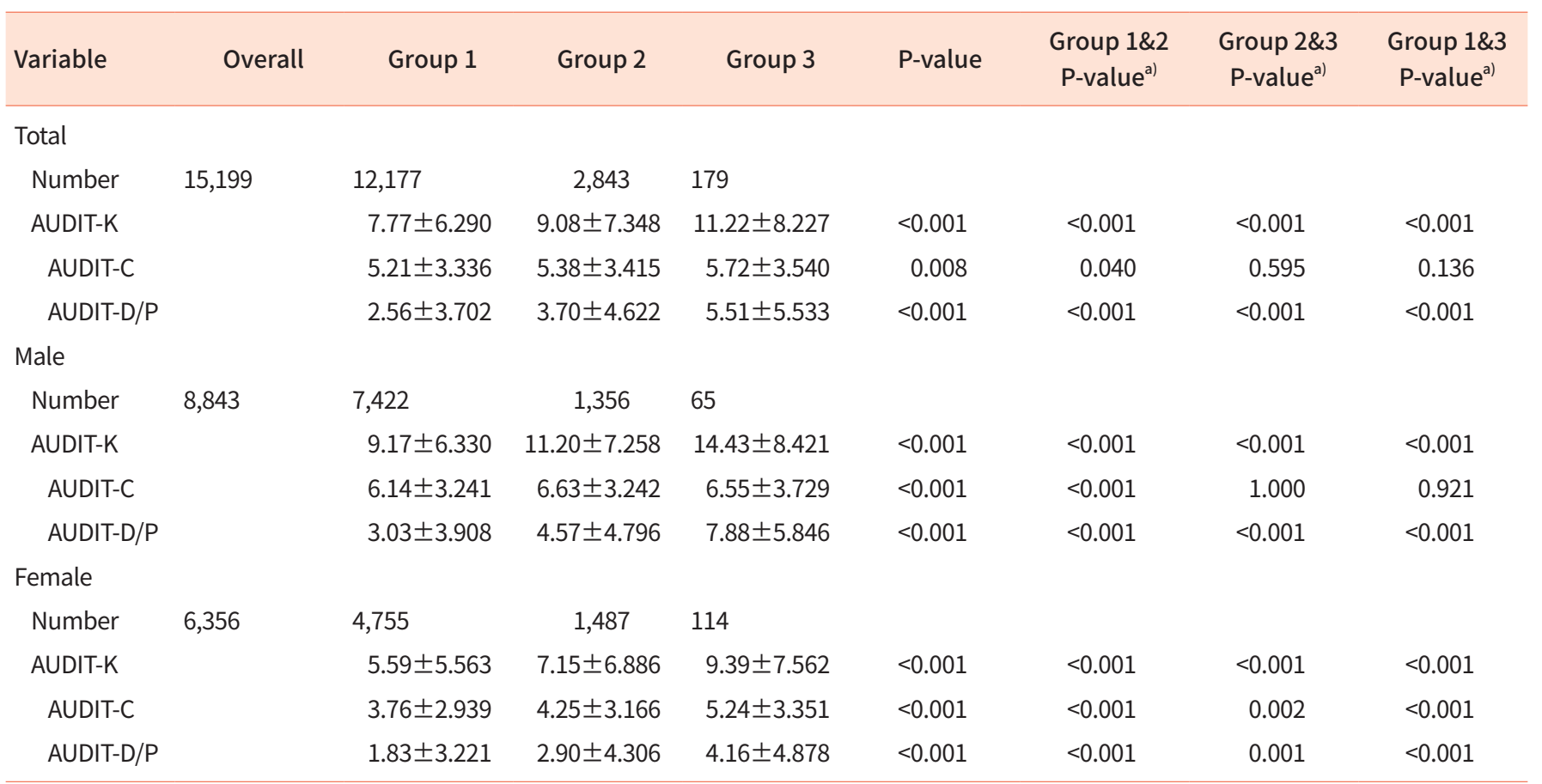

Values are presented as mean \pm standard deviation. Group 1: participants who had no suicidal ideation and had not attempted suicide; Group 2: participants who had suicidal ideation and had not attempted suicide; Group 3: participants who had suicidal ideation and had attempted suicide.

AUDIT-K, Alcohol Use Disorders Identification Test-Korea; AUDIT-C, alcohol consumption score; AUDIT-D/P, alcohol dependent/related problem score.

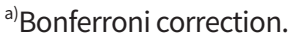




\section{Characteristics related to drinking}

The overall score (AUDIT-K) and item scores (AUDIT-C, AUDIT-D/P) for drinking scales were compared between the groups, and all scores were significantly different, indicating that as alcohol use problem increased, suicide severity also increased. With regard to the AUDIT-C, no statistically significant difference between Group 2 and Group 3 was observed in the post hoc analysis $(P=0.595)$, while a statistically significant difference for all three groups was observed for the AUDIT-D/P in the post hoc analysis (Table 3).

Meanwhile, alcohol consumption quantity and behavior are different according to sex [23]. When the overall scores and item scores were compared for all groups according to sex, all scores increased from Group 1 to Group 3. For male participants $(n=8,843)$, the difference in the AUDIT-C items between Group 2 and Group 3 was statistically non-significant $(P=1.000)$, while those for the AUDIT-D/P were all statistically significant $(P<0.001)$ in post hoc analyses. For female participants $(n=6,356)$, the differences in the overall scores and item scores were statistically significant for all groups in the post hoc analyses (Table 3).
Also, the overall scores and item scores were compared by dividing the participants in each group by age to examine whether there was a difference according to age. Scores for all ages were significantly different between the groups, except for AUDIT-C scores for participants in their 20s $(P=0.553)$, among whom $(n=2,718)$, there was no significant difference in any scores between Group 2 and Group 3 according to post hoc analyses. Additionally, the difference in the AUDIT-C scores for participants in their 30s $(n=5,566)$ was not statistically significant between Group 2 and Group 3 in post hoc analyses $(P=0.066)$, whereas the difference in AUDIT-D/P was statistically significant $(P=0.007)$. Similarly, the difference in the AUDIT-C scores for participants in their 40s or over $(n=$ 6,915 ) was not statistically significant between Group 2 and Group 3 in post hoc analyses either $(P=1.000)$, whereas the difference in the AUDIT-D/P across groups was statistically significant $(P<0.001)$ (Table 4).

\section{DISCUSSION}

This study analyzed various factors that may influence sui-

Table 4. AUDIT-K specific factor analysis by age group

\begin{tabular}{|c|c|c|c|c|c|c|c|c|}
\hline & Overall & Group 1 & Group 2 & Group 3 & P-value & $\begin{array}{c}\text { Group 1\&2 } \\
\text { P-value }^{\text {a) }}\end{array}$ & $\begin{array}{c}\text { Group 2\&3 } \\
\text { P-value }\end{array}$ & $\begin{array}{c}\text { Group } 1 \& 3 \\
\text { P-value }^{\text {a) }}\end{array}$ \\
\hline \multicolumn{9}{|l|}{$20 \mathrm{~s}$} \\
\hline Number & 2,718 & 2,145 & 534 & 39 & & & & \\
\hline AUDIT-K & & $8.04 \pm 6.049$ & $9.30 \pm 7.425$ & $9.16 \pm 8.803$ & $<0.001$ & $<0.001$ & 1.000 & 0.860 \\
\hline AUDIT-C & & $5.36 \pm 3.053$ & $5.43 \pm 3.288$ & $4.87 \pm 3.618$ & 0.553 & 1.000 & 0.859 & 1.000 \\
\hline \multicolumn{9}{|l|}{$30 \mathrm{~s}$} \\
\hline Number & 5,566 & 4,300 & 1,190 & 76 & & & & \\
\hline AUDIT-K & & $7.75 \pm 6.400$ & $8.73 \pm 7.158$ & $11.08 \pm 7.377$ & $<0.001$ & $<0.001$ & 0.008 & $<0.001$ \\
\hline AUDIT-C & & $5.11 \pm 3.299$ & $5.19 \pm 3.349$ & $6.09 \pm 3.082$ & 0.031 & 1.000 & 0.066 & 0.031 \\
\hline AUDIT-K & & $7.69 \pm 6.293$ & $9.34 \pm 7.499$ & $12.60 \pm 8.673$ & $<0.001$ & $<0.001$ & $<0.001$ & $<0.001$ \\
\hline AUDIT-C & & $5.23 \pm 3.461$ & $5.56 \pm 3.534$ & $5.77 \pm 3.944$ & 0.007 & 0.010 & 1.000 & 0.637 \\
\hline AUDIT-D/P & & $2.46 \pm 3.566$ & $3.78 \pm 4.636$ & $6.83 \pm 5.749$ & $<0.001$ & $<0.001$ & $<0.001$ & $<0.001$ \\
\hline
\end{tabular}

Values are presented as mean \pm standard deviation. Group 1: participants who had no suicidal ideation and had not attempted suicide; Group 2: participants who had suicidal ideation and had not attempted suicide; Group 3: participants who had suicidal ideation and had attempted suicide.

AUDIT-K, Alcohol Use Disorders Identification Test-Korea; AUDIT-C, alcohol consumption score; AUDIT-D/P, alcohol dependent/related problem score.

a) Bonferroni correction. 
cidal ideation and suicide attempt in the working population of Korea and confirmed that alcohol dependence and problematic behavior after alcohol consumption play key roles in suicide attempts. Many studies have focused on the relationship between alcohol use and suicide, but no study has separated the drinking problem by alcohol consumption quantity from related problematic behavior and individually assessed their risk for suicide attempt. This is also the first largescale study that examines the relationship between alcohol use and suicide attempt in the working population which includes relatively a large number of participants who attempted suicide, thus highlighting the importance of identifying high-risk groups for suicide among employees.

It was found that alcohol dependence and related problematic behavior has a strong connection from suicidal thoughts to actual suicide attempts. This correlation was common in both sexes and across all age groups above 30 years old. Subcategories of the AUDIT-D/P related to alcohol dependence and problematic behavior include impaired control over drinking, malfunction, morning drinking, feelings of guilt, blackouts, alcohol-related injuries, and the concern of others [13], which are indices for alcohol-related dependence and functioning in daily living. The items of the AUDIT-D/P represent different effects of frequent drinking in daily life, which may be caused by reduced executive function and can further deteriorate executive function. Thus, a prediction can be made that the AUDIT-D/P items would be significantly correlated with attempted suicide risk. Drinking has consistently been demonstrated in previous studies to induce deteriorated executive function such as cognitive function, psychomotor retardation, and response inhibition, not only in patients with alcohol use disorder (AUD) but also in the general population [24]. Suicide is associated with impairment of executive functions such as decision-making and impulse control [25], which are known to increase suicide risk in patients with depression [26,27]. Additionally, suicidal behavior in patients with AUD is strongly correlated with psychopathological symptoms of alcohol dependence, like poor impulse control [28]. Suicide attempts in these patients are less fatal and tend to be repetitive and are thus often collectively referred to as "deliberate self-harm syndrome" [29]. Accordingly, it can be predicted that individuals with suicidal ideation may have a high risk of future suicide attempts due to deteriorated judgment and difficulty with impulse control caused by executive function impairment after drinking. More accurate assessments and interventions are needed for drinkers with a high risk for suicide, considering the high possibility of recurrence of future suicide attempts [28].

Additional analyses to examine differences by sex showed that female participants exhibited an apparent correlation between past suicide attempt and drinking quantity, unlike male participants. This result may be attributed to alcohol tolerance differences between men and women. Men have higher total body water volume and higher levels of alcohol dehydrogenase than women and are less affected by drinking quantity [30]. These vulnerabilities must be taken into consideration when establishing preventive measures for suicide because consumption quantity has a greater effect on suicide attempt risk in women. This finding is consistent with a previous study that reported that females have a higher rate of intoxication and twice the BAC or higher than males at the time of committing suicide [6].

This study also included an analysis stratified by age, because age differences in alcohol consumption have been reported [31]. The result showed an increasing correlation between alcohol dependence and problematic behavior and suicide attempt with increasing age, which was in contrast to drinking quantity and frequency. The elderly are generally known to have lower rates of excessive drinking and higher rates of non-drinking compared with younger groups [31]. However, most of the participants in this study were assumed to be of relatively young age compared with other elderly studies because this study specifically targeted the working population. Therefore, the results are likely to be due to decreased metabolism by increased age, as change in drinking amount is not so significant within the working population.

The first limitation of this study is that a causal relationship between alcohol use and suicide could not be demonstrated because of the cross-sectional design. Second, the assessment of each risk factor was subjectively assessed and greatly simplified due to the self-reported survey. Third, the results may not be representative of the general working population or the general population because the study targeted subjects recruited from a few institutions. Moreover, the groups also differ in other psychopathologies such as depression and anxiety, and these interactions may have influenced suicidality.

Despite these limitations, this study offers several contributions and advantages. First, it is the first study to identify a correlation between alcohol use and suicide attempt based on analyses that distinguish between drinking amount/frequency and dependence/problematic behavior. Second, the validity and reliability of the self-reporting scales used in this 
study have been established in numerous previous studies and are currently used in various institutions. Third, compared with previous studies conducted on a relatively small number of subjects, this study recruited nearly 15,000 participants to obtain more objective and reliable results, thus enabling a more meaningful contribution to establishment of preventive measures for suicide. Fourth, excluding autopsy studies, this is the first study to focus on drinking in a large number of individuals who had actually attempted suicide. This study can be supplemented in the future by targeting a greater number of participants in the working population or the general population or by conducting analyses on detailed items related to alcohol consumption.

In conclusion, alcohol-related dependence and problematic behavior in the working population are strongly correlated with risk of attempting suicide. Therefore, an assessment of drinking-related factors is particularly crucial among various factors that can evaluate suicide risk. It is important to identify which people have a higher risk of attempting suicide during mental health examinations, and to emphasize the correlation between alcohol-related dependence and problematic behavior and suicide attempts during education on drinking and suicide prevention at the workplace. Consequently, the socioeconomic loss to corporations and society can be minimized if suicide attempts can be prevented. Moreover, social mechanisms should be provided to enable people to seek professional help more easily when they consider themselves at risk.

\section{CONFLICTS OF INTEREST}

No potential conflict of interest relevant to this article was reported.

\section{ORCID}

Won Young Choo

Ung Lee

Jae-Hyun Park

Young-Chul Shin

Mikyung Sim

Dong-Won Shin

Sang-Won Jeon

Min-Kyoung Kim

Sung Joon Cho
Kang-Seob Oh https://orcid.org/0000-0002-5054-6031 https://orcid.org/0000-0002-1656-9133 https://orcid.org/0000-0001-5860-7487 https://orcid.org/0000-0002-1884-4231 https://orcid.org/0000-0001-7918-644X https://orcid.org/0000-0001-9850-1898 https://orcid.org/0000-0001-6082-9599 https://orcid.org/0000-0002-7828-3296 https://orcid.org/0000-0002-5596-5665 https://orcid.org/0000-0001-6981-0931

\section{AUTHOR CONTRIBUTIONS}

Conception or design: WYC, SJC.

Acquisition, analysis, or interpretation of data: WYC, JHP, MS, SJC.

Drafting the work or revising: WYC, UL, YCS, KSO, DWS, SWJ, MKK, SJC.

Final approval of the manuscript: SJC.

\section{REFERENCES}

1. Korean Statistical Information Service. Suicide rates per 100,000 population [Internet]. Daejeon (KR): Korean Statistical Information Service; 2020 [cited 2021 May 21]. Available from: https://kosis.kr/eng/.

2. Organisation for Economic Co-operation and Development. Suicide rates (indicator) [Internet]. Paris (FR): Organisation for Economic Co-operation and Development; 2021 [cited 2021 May 21]. Available from: https://data.oecd.org/healthstat/suicide-rates.htm.

3. National Health Insurance Service. Socioeconomic cost analysis of major diseases for prioritizing health security policy, National Health Insurance Policy Research Institute of Korea National Health Insurance Service [Internet]. Wonju (KR): National Health Insurance Service; 2017 [cited 2021 May 21]. Available from: https://www.nhis.or.kr/ english/index.do.

4. Korea Ministry of Health and Welfare. Korea National Health and Nutrition Examination survey [Internet]. Cheongju (KR): Korea Ministry of Health and Welfare; 2020 [cited 2021 May 21]. Available from: https://knhanes.cdc.go.kr/knhanes/ eng/index.do.

5. World Health Organization. Global Information System on Alcohol and Health [Internet]. Geneva (CH): World Health Organization; 2020 [cited 2021 May 21]. Available from: https://www.who.int/gho/alcohol/en/.

6. Choi H, Seo JS, Pyo JY, Park YH. Correlation of alcohol intake with suicide in cases confirmed by legal autopsy. Korean J Leg Med 2012;36:63-7.

7. Powers RJ, Kutash IL. Alcohol, drugs, and partner abuse. In: Roy M, editor. The abusive partner: an analysis of domestic battering. New York (NY): Van Nostrand Reinhold; 1982. p. 39-75.

8. Brent DA, Baugher M, Bridge J, Chen T, Chiappetta L. Ageand sex-related risk factors for adolescent suicide. J Am Acad Child Adolesc Psychiatry 1999;38:1497-505.

9. Klimkiewicz A, Ilgen MA, Bohnert AS, Jakubczyk A, Wo- 
jnar M, Brower KJ. Suicide attempts during heavy drinking episodes among individuals entering alcohol treatment in Warsaw, Poland. Alcohol Alcohol 2012;47:571-6.

10. Sher L. Alcohol consumption and suicide. QJM 2006;99:5761.

11. Klonsky ED, Victor SE, Saffer BY. Nonsuicidal self-injury: what we know, and what we need to know. Can J Psychiatry 2014;59:565-8.

12. Pompili M, Goracci A, Giordano G, Erbuto D, Girardi P, Klonsky ED, et al. Relationship of non-suicidal self-injury and suicide attempt: a psychopathological perspective. J Psychopathol 2015;21:348-53.

13. Lee DK, Shin JK, Yun SM, Byun W. A reliability and validity study of the Korean version of the alcohol dependence scale in alcoholics. J Korean Acad Addict Psychiatry 2000; 4:30-7.

14. Bush K, Kivlahan DR, McDonell MB, Fihn SD, Bradley KA. The AUDIT alcohol consumption questions (AUDIT-C): an effective brief screening test for problem drinking. Ambulatory Care Quality Improvement Project (ACQUIP). Alcohol Use Disorders Identification Test. Arch Intern Med 1998;158:1789-95.

15. Bradley KA, McDonell MB, Bush K, Kivlahan DR, Diehr P, Fihn SD. The AUDIT alcohol consumption questions: reliability, validity, and responsiveness to change in older male primary care patients. Alcohol Clin Exp Res 1998;22: 1842-9.

16. Cho MJ, Kim KH. Use of the center for epidemiologic studies depression (CES-D) scale in Korea. J Nerv Ment Dis 1998; 186:304-10.

17. Yook SP, Kim ZS. A clinical study on the Korean version of Beck Anxiety Inventory: comparative study of patient and non-patient. Korean J Clin Psychol 1997;16:185-97.

18. Chang SJ, Koh SB, Kang D, Kim SA, Kang MG, Lee CG, et al. Developing an occupational stress scale for Korean employees. Korean J Occup Environ Med 2005;17:297-317.

19. Lee J, Shin C, Ko YH, Lim J, Joe SH, Kim S, et al. The reliability and validity studies of the Korean version of the Perceived Stress Scale. Korean J Psychosom Med 2012; 20:127-34.

20. Kearney CA, Drabman RS, Beasley JF. The trials of childhood: the development, reliability, and validity of the daily life stressors scale. J Child Fam Stud 1993;2:371-88.

21. Baek HS, Lee KU, Joo EJ, Lee MY, Choi KS. Reliability and validity of the Korean version of the connor-davidson resilience scale. Psychiatry Investig 2010;7:109-15.

22. Min SK, Kim KI, Suh SY, Kim DK. Development of the korean version of the World Health Organization Quality of Life Scale (WHOQOL). J Korean Neuropsychiatr Assoc 2000;39: 78-88.

23. Sadock BJ, Sadock VA. Alcohol-related disorder. In: Sadock BJ, Kaplan HI, Sadock VA, editors. Kaplan and Sadock's synopsis of psychiatry: behavioral sciences/clinical psychiatry. 10th ed. Philadephia (PA): Lippincott Williams \& Wilkins; 2011. p. 1472.

24. Houston RJ, Derrick JL, Leonard KE, Testa M, Quigley BM, Kubiak A. Effects of heavy drinking on executive cognitive functioning in a community sample. Addict Behav 2014; 39:345-9.

25. Onat M, Emiroglu NI, Baykara B, Ozerdem A, Ozyurt G, Ozturk $Y$, et al. Executive functions and impulsivity in suicide attempter adolescents with major depressive disorder. Psychiatr Clin Psychopharmacol 2019;29:332-9.

26. Bredemeier K, Miller IW. Executive function and suicidality: a systematic qualitative review. Clin Psychol Rev 2015; 40:170-83

27. Pu S, Setoyama S, Noda T. Association between cognitive deficits and suicidal ideation in patients with major depressive disorder. Sci Rep 2017;7:11637.

28. Lee J, Chang SJ, Kim H, Kim MH, Ahn JS, Park KC, et al. Prolonged risk of suicide reattempts in patients with alcohol use disorder and acute alcohol use: a register-based follow-up study (2010-2015). Psychiatry Investig 2019;16: 145-53.

29. Pennel L, Quesada JL, Begue L, Dematteis M. Is suicide under the influence of alcohol a deliberate self-harm syndrome? An autopsy study of lethality. J Affect Disord 2015; 177:80-5.

30. Kim SJ. Drinking experiences of women alcoholics: a feminist approach. J Korean Acad Psychiatr Ment Health Nurs 2006;15:362-74.

31. Shaw BA, Krause N, Liang J, McGeever K. Age differences in long-term patterns of change in alcohol consumption among aging adults. J Aging Health 2011;23:207-27. 